\title{
Design and Expected Performance of a Fast Neutron Attenuation Probe for Light Element Density Measurements
}

\author{
M. Sweany ${ }^{\mathrm{a}, *}$, P. Marleau ${ }^{\mathrm{a}}$ \\ ${ }^{a}$ Sandia National Laboratory, Livermore, CA 94550, USA
}

\begin{abstract}
We present the design and expected performance of a proof-of-concept 32 channel material identification system. Our system is based on the energy-dependent attenuation of fast neutrons for four elements: hydrogen, carbon, nitrogen and oxygen. We describe a new approach to obtaining a broad range of neutron energies to probe a sample, as well as our technique for reconstructing the molar densities within a sample. The system's performance as a function of time-of-flight energy resolution is explored using a Geant4-based Monte Carlo. Our results indicate that, with the expected detector response of our system, we will be able to determine the molar density of all four elements to within a 20-30\% accuracy in a two hour scan time. In many cases this error is systematically low, thus the ratio between elements is more accurate. This degree of accuracy is enough to distinguish, for example, a sample of water from a sample of pure hydrogen peroxide: the ratio of oxygen to hydrogen is reconstructed to within $8 \pm 0.5 \%$ of the true value. Finally, with future algorithm development that accounts for backgrounds caused by scattering within the sample itself, the accuracy of molar densities, not ratios, may improve to the 5-10\% level for a two hour scan time.
\end{abstract}

Keywords: neutron time-of-flight, material identification, explosives detection

\section{Introduction}

\subsection{Material identification techniques using neutrons}

Since the late 1980's, renewed interest in screening capabilities has resulted in significant investment in Thermal Neutron Analysis (TNA) and Fast Neutron Analysis (FNA) techniques as a complement to $\mathrm{x}$-ray techniques. Because $\mathrm{x}$-ray devices are only sensitive to variations in density, they do not provide effective material identification for light elements. In TNA, gamma particles resulting from a handful of thermal neutron capture reactions in materials of interest are used as a means of identification. FNA measures either the gamma rays from nuclei activated by a neutron beam, or the energy-dependence of an attenuated neutron beam. Several groups have demonstrated elemental imaging capabilities, but differ in their method of obtaining a range of neutron probe energies (see [1] for a review). Pulsed Fast Neutron Transmission Spectroscopy (PFNTS) employs the Time-of- Flight technique combined with sophisticated high-flux, pulsed accelerator technology to determine neutron energies $[2,3]$. Another method for obtaining a range of probe energies described in [4] involves accelerating deuterium or protons onto a deuterium or lithium target: neutrons are produced with energies dependent on the angle between the beam and detectors. This method requires multiple measurements at different orientations to obtain a range of energies, rather than a simultaneous measurement over all energies, increasing scan times. More recently, [5] used deuterium on a boron target to obtain several discrete neutron energies as a sample probe. The transmitted flux at each of these energies, measured by the energy deposited in liquid scintillator detectors, was used to inform the hydrogen content in samples. Finally, the CSIRO/NUTECH detector [6] uses $14.1 \mathrm{MeV}$ neutrons from a (d-T) source in combination with $6 / 3 \mathrm{MeV}$ $\mathrm{x}$-rays from a linear accelerator to determine both the average material density and composition of materials. The ratio of neutron and x-ray penetration, $R$, is indicative of the material present, and impressive 2D images have been obtained with conveyor belt speeds of $1-2$ $\mathrm{m} / \mathrm{min}$. However for both illicit drugs and explosives, the discrimination parameter $R$ has a significant overlap with common materials, causing a high false alarm rate.

*Corresponding author: msweany@sandia.gov 


\subsection{Light element reconstruction with fast neutron at- tenuation}

Our system is a neutron time-of-flight spectrometer consisting of two detector planes, designed to measure the unique attenuation spectrum of light elements. Specifically, the elements carbon, nitrogen and oxygen have many resonances in the total neutron cross section in the 1-10 MeV range, shown in Figure 1. Given sufficient energy resolution and a wide-range of energies to probe a sample, the attenuation spectrum can be used to uniquely determine the molar fractions of these elements contained in the sample. Although hydrogen does not have resonances, the energy dependance of its cross section does enable its molar fraction to be determined as well. The fraction of the aforementioned four elements can in many cases uniquely identify the material in question [1].

We create a range of probe energies with a monoenergetic neutron source by down-scattering in the forward detector plane of the system. A neutron from a $\mathrm{d}-\mathrm{T}$ generator elastically interact in the forward detector plane, depositing energy to start a time-of-flight clock. For a single interaction, the energy of the neutron after this interaction (the probe energy) is dependent on the scattering angle, $\theta$ :

$$
E_{i}=\frac{14.1 \mathrm{MeV}}{1+\tan ^{2} \theta}
$$

The neutron then passes through the sample region to the back detector plane, depositing energy and stopping the time-of-flight clock. The energy probing the sample is then calculated through time-of-flight with the distance between detector centers and measured time ( $r$ and $t$, respectively) between the two detector hits:

$$
E=\frac{m}{2}\left(\frac{r}{t}\right)^{2}
$$

While the energy deposited in the forward detector 107 plane is also a handle on the energy as it passes through 108 the sample, the resolution is not as good as that deter- 109 mined by time-of-flight. Because we are using time- 110 of-flight to directly calculate the scattered neutron's en- 111 ergy, we are able to remove the assumption that a sin- 112 gle interaction occurred in the forward detector plane. ${ }_{113}$ After many events over a range of scattering angles a 114 spectrum is obtained. By comparing spectra with and 115 without a sample present, an energy dependent attenua- 116 tion is obtained. A two-detector version of this system 117 is described in [8]: the goal of the work described here 118 is to scale to a larger system for faster scan times, and 119 to develop a model which will be validated with data in 120

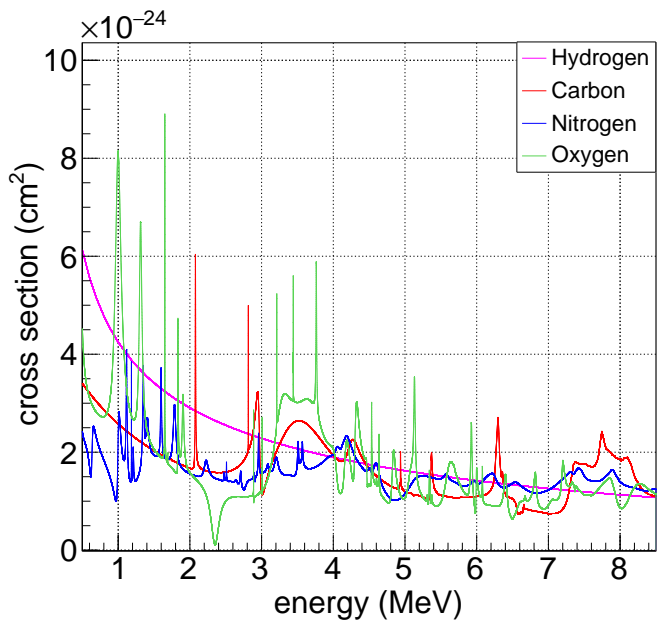

Figure 1: Left: The total neutron interaction cross section spectra for Hydrogen, Carbon, Nitrogen, and Oxygen, from ENDF/B-VII.0 [7].

the future. This model will be used to estimate the limits of this method given variables such as acquisition time, and timing and position resolution. Future studies will focus on room return backgrounds and how the number of detectors in the system effects reconstruction errors and background levels.

In Section 2 the Geant4-based Monte Carlo model is described, including geometry, event generation, and detector response. An evaluation of the detector system's performance is discussed in Section 3: although there is the potential for imaging capabilities with future algorithm development, in this work we evaluate material reconstruction alone. Finally, future work is discussed in Section 4.

\section{Geant4-based detector model}

Below we describe the geometry, event generation, and processing for these simulations. In the following, an event is denoted as any interaction resulting from one call to the primary generator class of Geant4: in this case, a call to the primary generator class results in one primary particle released into the world volume. To avoid confusion, what is commonly described as a detector event, resulting from e.g. energy deposited in scintillator, is referred to as a deposition. For all simulations in this work, Geant4 [9] version 10.01 is used with the QGSP_BERT_HP physics list. The goal of these simulations is to understand the effects of position and timing resolution of the individual detectors on the reconstructed elemental molar densities for a range of ma- 
terials, as well as to understand and account for effects such as scattering within the sample.

Our system is 32 channels, each consisting of a $3 \times 3$ inch right cylindrical aluminum cell, 0.1 inches thick on the top and 0.045 inches thick on the rounded side, containing liquid scintillator (EJ-309) and painted with a highly reflective coating (EJ 520). Coupled to each cell is a 3-inch PMT (Electron Tubes model 9821KB) shielded from the earth's magnetic field with mu-metal casing: according to the manufacturer [10], the typical electron transit time spread is $2.2 \mathrm{~ns}$ FWHM, and the single electron width is $3.2 \mathrm{~ns}$ (FWHM). The timing of our system is therefore limited to a standard deviation of approximately $1 \mathrm{~ns}$. It is feasible that we could reach this limit using a $500 \mathrm{MHz}$ digitizer, so for these studies we explore performance with timing resolutions of 2.5, 162 $1,0.5$, and $0 \mathrm{~ns}$ in order to span the range from worst 163 case scenario, likely scenario, likely improvement with 164 upgraded PMTs, and ideal. For the position, the sys- 165 tem is limited by the size of the cells and the probability 166 of neutron interactions within each of them. Because 167 the interaction position is geometry dependent, the in- 168 teraction position for each cell is approximated by con- 169 volving the average simulated true interaction position distribution.. The specifics of this characterization are 170 described in Section 2.3.

We calculate the error on time-of-flight energy, $E_{i}$, as: ${ }_{172}$

$$
\begin{aligned}
\sigma_{E} & =\sqrt{\left(\frac{\partial E}{\partial x}\right)^{2} \sigma_{x}^{2}+\left(\frac{\partial E}{\partial z}\right)^{2} \sigma_{z}^{2}+\left(\frac{\partial E}{\partial t}\right)^{2} \sigma_{t}^{2}} \\
\frac{\sigma_{E}}{E} & =2 \sqrt{\left(\frac{1}{r}\right)^{2} \sigma_{x}^{2}+\left(\frac{1}{t}\right)^{2} \sigma_{t}^{2}}
\end{aligned}
$$

where $r$ and $t$ are the distance and time separation of two neutron depositions, and $\sigma_{x}$ and $\sigma_{t}$ are the standard deviation of the position and time, as discussed above. The position resolution is ill-defined in this case, as we have characterized the overall resulting energy resolution, $f(E)$, due to the average position response for each individual detector. Equation 4 becomes:

$$
\frac{\sigma_{E}}{E}=\sqrt{\left(\frac{f(E)}{E}\right)^{2}+4\left(\frac{1}{t}\right)^{2} \sigma_{t}^{2}}
$$

\subsection{Geometry description}

As described above, our system consists of 32 channels of liquid scintillator cells. The cells are arranged in two 16 cell planar arrays, with the neutron generator rotated in a partial ring to obtain a range of scattering angles. The geometry is illustrated in Figure 2.

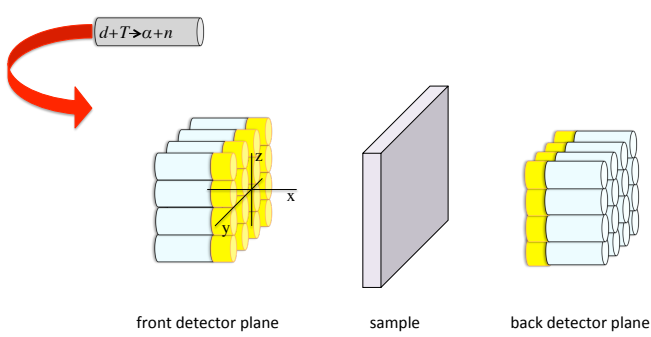

Figure 2: A rendering of the detector geometry: the liquid scintillator, shown in yellow, is separated by $150 \mathrm{~cm}$ center-to-center. The d-T generator (red arrow) sits at $+50 \mathrm{~cm}$ in the $\mathrm{z}$ direction and subtends a circle with radius $50 \mathrm{~cm}$ in the $\mathrm{x}-\mathrm{y}$ quadrant.

In our model, only the scintillator is included: we have assumed that scattering in assembly and PMT components are small effects compared to the bulk scintillator. Although material between the generator and the scintillator in the front detector plane may effect the energy spectrum, this effect will be essentially equal in the empty and sample datasets and not alter the attenuation spectrum.

\subsection{Event generation}

For increased simulation efficiency, we only generate neutrons with trajectories that will pass through the front detector plane. The generator position is confined to a $50 \mathrm{~cm}$ radial distance (in $\mathrm{x}$ and $\mathrm{y}$ ) from the center of the front detector plane, and $50 \mathrm{~cm}(\mathrm{z})$ above the plane. The angle along the circle is sampled uniformly, but restricted to one quadrant (in $x$ and $y$ ). Neutron trajectories are sampled isotropically, and rejected if they do not pass through the front detector plane. A separate simulation, which does not reject any trajectories, was used to calculate the average solid angle for dwell time calculations: 42,200 events out of 5 million neutrons hit the front detector plane, yielding an average solid angle of $0.844 \%$ of $4 \pi$. For a generator output of $1 \mathrm{e} 7$ neutrons per second, one million simulated events generated in this fashion is equivalent to 11.6 seconds of experimental acquisition time.

The energy of the neutron is determined by the orientation of the generator and the neutron trajectory:

$$
\sqrt{E}=\frac{-b+\sqrt{b^{2}-4 a c}}{2 a}
$$

with

$$
\begin{aligned}
a & =\frac{m_{\alpha}+m_{n}}{2}, \\
b & =-\sqrt{m_{d} m_{n} E_{d}} \cos \theta, \text { and }
\end{aligned}
$$




$$
c=\frac{-m_{\alpha} Q+E_{d}\left(m_{\alpha}-m_{d}\right)}{2},
$$

where $\theta$ is the angle between the generator's orientation and the neutron emission trajectory, $m_{n}, m_{d}$ and $m_{\alpha}$ are the mass of the neutron, deuteron, and alpha particle, respectively. $E_{d}$ is the energy of the deuteron, which is dependent on the operating parameters of the tube. Finally, $Q$ is the energy released in the $d+T \rightarrow \alpha+n$ interaction, $17.590 \mathrm{MeV}$.

Although the geometrical efficiency of our desired event has been improved by sampling only trajectories that hit the first detector plane, the probability that those neutrons will subsequently pass through the sample and interact in the second detector plane is still quite low. Because effects such as multiple depositions in the front detector plane are of interest, we do not perform further variance reduction methods. We do, however, restrict events that are processed further by only storing events that result in a deposition in the second detector plane. The position, time, deposited energy, and track length of all protons undergoing hadronic ionization are stored, along with the position, time, and kinetic energy of all neutron elastic collisions. The analysis toolkit ROOT [11] is used to store events in a TTree, which is then processed with a stand-alone $\mathrm{C}++$ executable that also uses many ROOT classes to further analyze the data.

\subsection{Detector response and event processing}

Detector hits are constructed by first sorting all par- 24 ticle depositions in an event by particle type. Proton 245 depositions are quenched using Birk's law (with $k_{b}=246$ $0.0123 \mathrm{~cm} / \mathrm{MeV}$ ) before being added to the total hit en- 247 ergy. Only depositions which occur within $5 \mathrm{~ns}$ of each 248 other in an individual detector segment are included in 249 a detector hit. The position of each deposition is de- 250 termined as the energy weighed centroid of the detector 251 hit, and the time is the first deposition within a detec- 252 tor. The time and position of the detector hit are later ${ }_{253}$ convoluted with a Gaussian response to reflect the elec- 254 tronics time response and average deposition location, 255 respectively. In experimental data, neutron activation is 256 expected to occur producing an accidental gamma back- 257 ground. We will use pulse-shape discrimination along ${ }_{258}$ with the energy deposited in the front plane to calculate 259 an expected time-of-flight window to reduce this back- 260 ground. The level of this background is expected to be 261 dependent on the surrounding environment, and will be 262 studied more extensively in experimental data. For this 263 work, deposition resulting from gamma interactions are 264 not included.

The events are processed to search for multiple de- 266 tector hits: we require at least one detector hit in both ${ }_{267}$

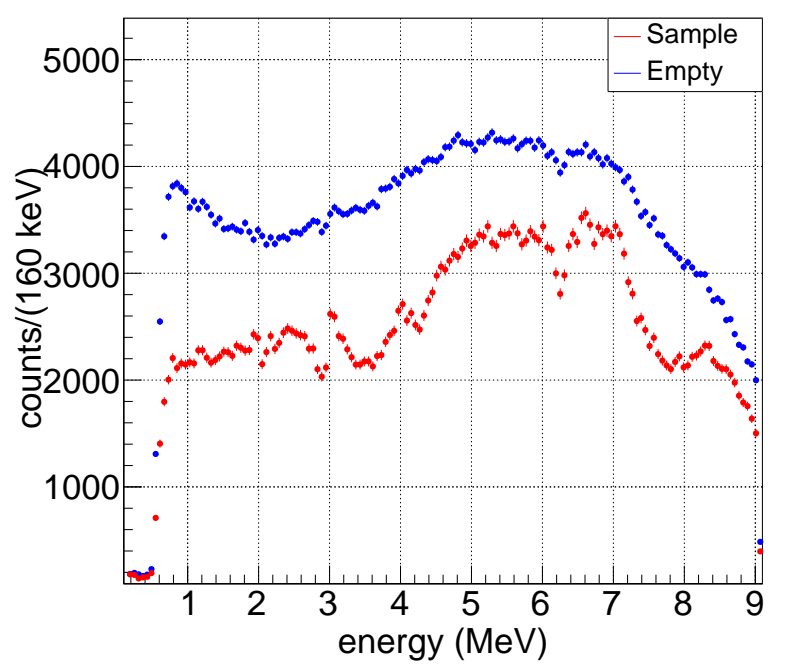

Figure 3: The time-of-flight energy spectrum with no sample present (blue) and a 2-cm thick graphite sample (red), for a two hour simulated run on graphite. The empty set is 5.5 hours, but has been scaled by the sample dwell time. Statistical error bars are shown. This time-offlight spectrum uses the true interaction position and time.

the near and far detector planes, and search for up to ten detector hits in an event. We require the hit energy in the far detector plane to be greater than $500 \mathrm{keVee}$. The true time-of-flight energy is then calculated using the detector hit time and position of the last deposition in the front detector plane and the detector hit time and position of the first deposition in the back detector plane. The attenuated energy spectrum is then calculated as the negative log ratio of the sample time-of-flight spectrum to the empty dataset. An example time-of-flight energy spectrum constructed with the true interaction positions and times is shown in Figure 3: the spectrum for both a $2 \times 30.48 \times 30.48 \mathrm{~cm}$ graphite target and no sample present is shown.

The position and time of each hit are then convoluted with a detector response. In the case of timing, the time is convoluted with a Gaussian of width 0.5 , 1 , or 2.5 ns. Figure 4 shows the standard deviation of the timeof-flight energy as a function of true energy for two depositions $r=150 \mathrm{~cm}$ apart and for a range of timing responses. For the position response, the empty dataset is used to characterize the average position response for a range of generator positions. These response functions are randomly sampled to determine the deposition location in each cell: for each event, the position distribution is sampled 100 times for the near and far detector cell, and a weighted entry (by 0.01) is input into the time-of- 


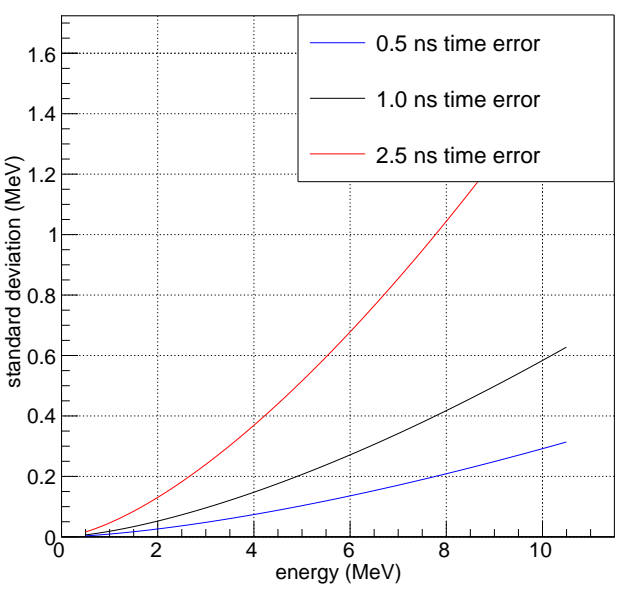

Figure 4: The standard deviation for a range of timing widths, with no detector response applied.

flight energy spectrum. After this position convolution is applied, the width of the time-of-flight energy spectrum is characterized as a function of energy: $f(E)$ is shown in Figure 5, along with linear fit parameters and the deviation from the fit. Our system's overall expected energy resolution of the time-of-flight energy follows the blue curve in Figure 6.

\section{System performance}

\subsection{Reconstruction method}

The attenuated energy spectrum is used to reconstruct the molar concentration of each of the four light elements considered. Binned cross section data for each of the four elements is filled with interpolated data from the ENDF/B-VII.0 database [7]: the bin width was chosen to be fine enough to capture all resonance peaks. A Gaussian convolution with the expected energy resolu- 295 tion from Equation 5 is performed, then the cross sec- 296 tion is integrated over the wider bin range of the atten- 297 uated energy spectrum: the average cross section cal- 298 culated in this manner is denoted $\langle\sigma\rangle$. Beer's law is used to calculate the expected number of transmitted particles in a particular energy bin, $e$, and for a particular detector pair, $d$ :

$$
\lambda_{e, d}=N_{e, d}^{e m p t y} e^{-N_{A} l_{d} \sum_{i=H C N O} M_{i}<\sigma_{i, e}>},
$$

where $N_{e, d}^{e m p t y}$ is the measured number of counts with- 303 out the sample present. The molar fraction of the 304 sample, $M_{i}$, for each element $i=\mathrm{HCNO}$, is in units of 305

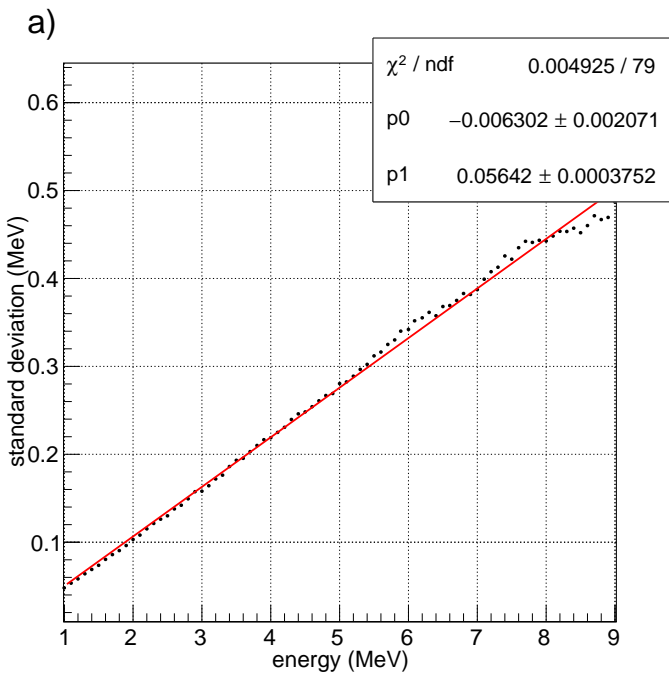

b)

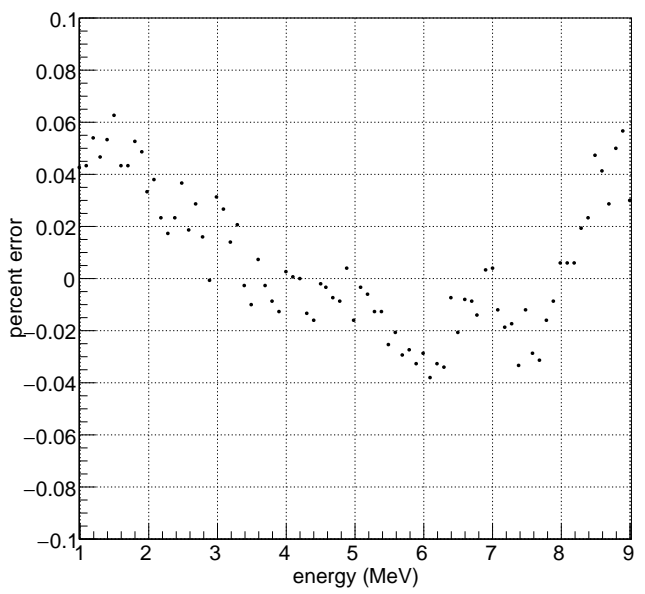

Figure 5: a) The standard deviation of the time-of-flight energy spectrum after applying the position convolution, b), the deviation (percent difference) from a linear fit.

moles $/ \mathrm{cm}^{3}$ due to the factor of Avogadro's number, $N_{A}$, included. Finally, $l_{d}$ is the detector-dependent path length through the sample. Using this expected value, a likelihood function is constructed:

$$
\mathcal{L}=\prod_{e=1, . ., n_{e}} \prod_{d=1, . ., n_{d}} e^{-n_{e, d}} \frac{n_{e, d}^{\lambda_{e, d}}}{\lambda_{e, d} !}
$$

where $n_{e, d}=N_{e, d}^{\text {sample }}$ is the measured number of counts in energy bin $e$ and for detector pair $d$ with the sample present. The energy range included is from the threshold energy on the far detector plane $(0.5 \mathrm{MeV})$ to the point at which there are no longer strong resonance peaks $(9 \mathrm{MeV})$. The MINUIT [12,13] package 


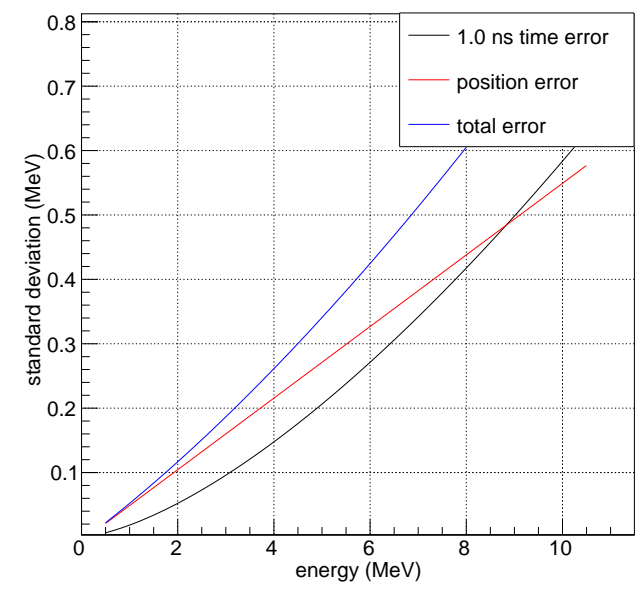

Figure 6: The standard deviation due to $1.0 \mathrm{~ns}$ timing convolution (black), position convolution (red) and the two combined (blue).

included in ROOT is used to minimize the negative loglikelihood function:

$$
\log \mathcal{L}=\sum_{e=0}^{n_{e}} \sum_{d=0}^{n_{d}} n_{e, d} \log \left(\lambda_{e, d}\right)-\lambda_{e, d}
$$

resulting in the overall elemental material composition of the sample in which the predicted values $\lambda_{e, d}$ are most likely to have produced the measured values $n_{e, d}$. We first call the SIMPLEX algorithm as a seed to the MIGRAD algorithm, and use the error matrix from MIGRAD to calculate the accuracy of the free parameters. In order to avoid the issues associated with parameter errors near a limit [13], which potentially occurs whenever an element is absent from a sample, the parameters are given no limits. The error definition is set to 0.5 , for one-standard-deviation values with a negative loglikelihood function, and the parameter step size is $0.01{ }^{347}$ $\mathrm{M} / \mathrm{cm}^{3}$. In the case of a true molar fraction of zero, the ${ }^{348}$ percent error is tabulated as n/a. When an initial fit re- 349 constructs values close to zero, the parameter is fixed to ${ }^{350}$ zero and the reconstruction is repeated.

Currently, effects of floor scattering and scattering ${ }^{352}$ within the sample are neglected. Scattering off of the ${ }^{353}$ floor in experimental data can be accounted for in the ${ }^{354}$ reconstruction by including an energy and detector-pair ${ }^{355}$ dependent experimental measurement in which direct ${ }^{356}$ paths between the two detector planes are blocked by ${ }^{357}$ a thick polyethylene block, and subtracting this value ${ }^{358}$ from the the number of attenuated particles:

$$
\lambda_{e, d}=\left(N_{e, d}^{e m p t y}-N_{e, d}^{\text {poly }}\right) e^{-N_{A} l_{d} \sum_{i=H C N O} M_{i} \sigma_{i, e}} .
$$

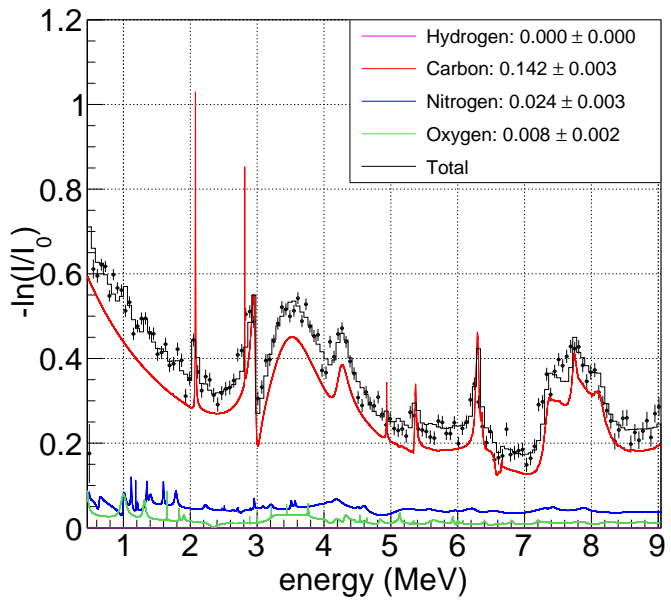

Figure 7: The attenuated energy spectrum for a $2 \times 30.48 \times 30.48$ graphite sample including only events which did not scatter in the sample and no detector response. The reconstructed molar fractions for all four elements are shown in the legend, and the corresponding contribution to the total attenuated spectrum is plotted.

Accounting for scattering within the sample is more complicated, and would involve adding a term in which the attenuated contributions from the entire sample are scattered into each detector pair according to the angular dependence of the cross section, which is dependent on the nuclei in which the neutron scattered off of. In other words, the sample would be broken into voxels, and the current estimate of each voxel's contents used to redistribute a portion of the attenuated particles into other detector pairs. The amount redistributed would be based on the angular cross section, energy, and path length through the sample into the particular voxel.

For the majority of results in this study we do not attempt to account for this effect, rather the effect of scattering is eliminated by taking only coincident hits in which no energy was lost in the sample. For the experimental campaign, scattering within the sample will be accounted for by subtracting the detector-dependent energy spectrum of each detector pair due to scattering alone from the total energy spectrum. Figure 7 shows the attenuated spectrum with no detector timing or position response, along with the total spectrum given the reconstructed values and the contribution from each element. Figure 8 shows the attenuated spectrum with 1.0 ns timing and position response applied; the cross sections used in the reconstruction have been convoluted with the expected error from Equation 5. Note that the total attenuated spectrum is shown for visual purposes 


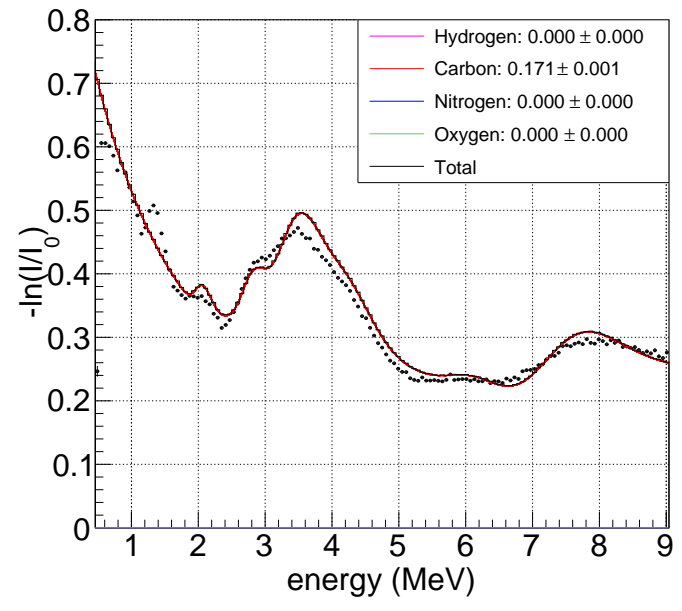

Figure 8: The attenuated energy spectrum for a $2 \times 30.48 \times 30.48$ graphite sample with the expected detector response applied and no sample scattering background. The reconstructed molar fractions for all four elements are shown in the legend, and the corresponding contribution to the total attenuated spectrum is plotted.

only, and that the reconstruction is performed on the attenuation spectrum of each detector pair.

\subsection{Results}

Several different samples are used to evaluate the reconstruction errors. We evaluated the system for different energy resolutions resulting from a range of tim- 403 ing resolutions, and also for a variety of acquisition 404 times. For all results below, the empty dataset is higher 405 statistics: at least four hours real time with a $1 \mathrm{e} 7 \mathrm{n} / \mathrm{s} 406$ generator output. Both the empty dataset and sample 407 dataset are processed in the same manner, e.g. the same ${ }_{408}$ time smearing is used. We study five samples, each 409 with dimensions $2 \times 30.48 \times 30.48 \mathrm{~cm}$ : ammonium ni- 410 trate, graphite, high-density polyethylene (HDPE), hy- ${ }_{411}$ drogen peroxide, and water. Graphite was chosen to 412 study the energy resolution and statistical effects be- 413 cause it consists of only one element, carbon. HDPE 414 was chosen to characterize performance with the addi- 415 tion of hydrogen, which does not have resonances but ${ }_{416}$ is energy dependent. Ammonium Nitrate was chosen ${ }_{417}$ because it consists of nitrogen and hydrogen, and nitro- ${ }_{418}$ gen's many tight resonances could be mistaken for sta- 419 tistical effects. Finally, hydrogen peroxide and water are ${ }_{420}$ of interest because they contain the same elements, hy- ${ }_{421}$ drogen and oxygen, but with different fractions: there- ${ }_{422}$ fore this will evaluate our ability to not only identify ${ }_{423}$ elements within a sample, but to quantify their concen- ${ }^{424}$ trations.
The molar density of an element $X$ is calculated for a sample by

$$
M_{X}=\frac{N_{X} \rho}{N_{H} m_{H}+N_{C} m_{C}+N_{N} m_{N}+N_{O} m_{O}},
$$

where $N_{H, C, N, O}$ is the number of atoms of each element, $m_{H, C, N, O}$ is the atomic mass of each element (multiplied by the molar mass constant), and $\rho$ is the mass density of the sample. For example, graphite, with a mass density of $2.08 \mathrm{~g} / \mathrm{cm}^{3}$ and consisting of only carbon, has a carbon molar density of

$$
M_{C}=\frac{1 * 2.09 \mathrm{~g} / \mathrm{c}^{3}}{1 * 12 \mathrm{~g} / \mathrm{mol}}=0.174 \mathrm{~mol} / \mathrm{cm}^{3} .
$$

Similarly, for water, the molar mass of hydrogen and oxygen are:

$$
\begin{aligned}
M_{H} & =\frac{2 * 1.0 \mathrm{~g} / \mathrm{cm}^{3}}{2 * 1.008 \mathrm{~g} / \mathrm{mol}+1 * 15.99 \mathrm{~g} / \mathrm{mol}} \\
& =0.111 \mathrm{~mol} / \mathrm{cm}^{3}
\end{aligned}
$$

and

$$
\begin{aligned}
M_{O} & =\frac{1 * 1.0 \mathrm{~g} / \mathrm{cm}^{3}}{2 * 1.008 \mathrm{~g} / \mathrm{mol}+1 * 15.99 \mathrm{~g} / \mathrm{mol}} \\
& =0.056 \mathrm{~mol} / \mathrm{cm}^{3},
\end{aligned}
$$

respectively. Table 1 includes the molar density of all samples included in this study, calculated in this fashion.

The results are included in Tables 2-7. Table 2 shows the reconstructed molar fractions for all four elements and the five samples in Table 1: for the results in this table, each sample dataset is two hours long, and no timing or position detector response is applied. The parameter error for each molar fraction is the result from MINUIT, and the value in parentheses are the percent error of the value compared to true values listed in Table 1 . The results in Table 2 indicate overall good agreement with the true values, with the lowest degree of accuracy from carbon, and the highest from hydrogen.

Next, the effects of detector position and timing response are studied for all samples. Tables 3 and 4 show the reconstructed molar fractions for all five samples with the position response only applied and both the detector and timing response applied, respectively. In general, the accuracy has improved compared to the case with no detector response (Table 2), presumably because statistical fluctuations have been mis-identified as resonances. 


\begin{tabular}{|l|c|c|c|c|c|}
\hline Sample & $\rho\left(\mathrm{g} / \mathrm{cm}^{3}\right)$ & Hydrogen $\left(\mathrm{M} / \mathrm{cm}^{3}\right)$ & Carbon $\left(\mathrm{M} / \mathrm{cm}^{3}\right)$ & Nitrogen $\left(\mathrm{M} / \mathrm{cm}^{3}\right)$ & Oxygen $\left(\mathrm{M} / \mathrm{cm}^{3}\right)$ \\
\hline \hline Ammonium Nitrate & 1.725 & 0.086 & 0.00 & 0.043 & 0.065 \\
Graphite & 2.09 & 0.00 & 0.174 & 0.00 & 0.00 \\
HDPE & 0.94 & 0.134 & 0.067 & 0.00 & 0.00 \\
Hydrogen Peroxide & 1.45 & 0.085 & 0.00 & 0.00 & 0.085 \\
Water & 1.00 & 0.111 & 0.00 & 0.00 & 0.056 \\
\hline
\end{tabular}

Table 1: The true molar concentrations for all samples studied.

\begin{tabular}{|l||c|c|c|c|}
\hline Sample & $\mathrm{H}\left(\mathrm{M} / \mathrm{cm}^{3}\right)(\%)$ & $\mathrm{C}\left(\mathrm{M} / \mathrm{cm}^{3}\right)(\%)$ & $\mathrm{N}\left(\mathrm{M} / \mathrm{cm}^{3}\right)(\%)$ & $\mathrm{O}\left(\mathrm{M} / \mathrm{cm}^{3}\right)(\%)$ \\
\hline \hline Ammonium Nitrate & $0.084 \pm 0.002(2.3)$ & $0.000 \pm 0.000(0.0)$ & $0.052 \pm 0.003(20.9)$ & $0.057 \pm 0.002(12)$ \\
Graphite & $0.000 \pm 0.000(0.0)$ & $0.142 \pm 0.003(18)$ & $0.024 \pm 0.003(\mathrm{n} / \mathrm{a})$ & $0.008 \pm 0.002(\mathrm{n} / \mathrm{a})$ \\
HDPE & $0.130 \pm 0.002(3.0)$ & $0.052 \pm 0.004(22)$ & $0.018 \pm 0.004(\mathrm{n} / \mathrm{a})$ & $0.000 \pm 0.000(0.0)$ \\
Hydrogen Peroxide & $0.082 \pm 0.002(3.5)$ & $0.014 \pm 0.003(\mathrm{n} / \mathrm{a})$ & $0.000 \pm 0.000(0.0)$ & $0.074 \pm 0.002(13)$ \\
Water & $0.111 \pm 0.002(0.0)$ & $0.000 \pm 0.000(\mathrm{n} / \mathrm{a})$ & $0.000 \pm 0.000(0.0)$ & $0.056 \pm 0.002(0.0)$ \\
\hline
\end{tabular}

Table 2: The reconstructed molar concentrations for all samples studied with no detector response applied (and no source scattering background). The dwell time for each case is two hours. The percent error from the true value is quoted in parentheses. The parameter errors are one standard deviation, from the error matrix of MINUIT.

\begin{tabular}{|l|c|c|c|c|}
\hline Sample & $\mathrm{H}\left(\mathrm{M} / \mathrm{cm}^{3}\right)(\%)$ & $\mathrm{C}\left(\mathrm{M} / \mathrm{cm}^{3}\right)(\%)$ & $\mathrm{N}\left(\mathrm{M} / \mathrm{cm}^{3}\right)(\%)$ & $\mathrm{O}\left(\mathrm{M} / \mathrm{cm}^{3}\right)(\%)$ \\
\hline \hline Ammonium Nitrate & $0.083 \pm 0.002(3.5)$ & $0.000 \pm 0.000(0.0)$ & $0.050 \pm 0.003(16)$ & $0.061 \pm 0.003(6.2)$ \\
Graphite & $0.000 \pm 0.000(0.0)$ & $0.172 \pm 0.001(1.1)$ & $0.000 \pm 0.000(0.0)$ & $0.000 \pm 0.000(0.0)$ \\
HDPE & $0.131 \pm 0.003(2.2)$ & $0.068 \pm 0.003(1.5)$ & $0.000 \pm 0.000(0.0)$ & $0.000 \pm 0.000(0.0)$ \\
Hydrogen Peroxide & $0.082 \pm 0.002(3.5)$ & $0.000 \pm 0.000(0.0)$ & $0.000 \pm 0.000(0.0)$ & $0.086 \pm 0.002(1.2)$ \\
Water & $0.106 \pm 0.002(4.5)$ & $0.000 \pm 0.000(0.0)$ & $0.000 \pm 0.000(0.0)$ & $0.060 \pm 0.002(7.1)$ \\
\hline
\end{tabular}

Table 3: The reconstructed molar concentrations for all samples studied with only the position response applied (and no source scattering background). The dwell time for each case is two hours. The percent error from the true value is quoted in parentheses. The parameter errors are one standard deviation, from the error matrix of MINUIT.

\begin{tabular}{|l||c|c|c|c|}
\hline Sample & $\mathrm{H}\left(\mathrm{M} / \mathrm{cm}^{3}\right)(\%)$ & $\mathrm{C}\left(\mathrm{M} / \mathrm{cm}^{3}\right)(\%)$ & $\mathrm{N}\left(\mathrm{M} / \mathrm{cm}^{3}\right)(\%)$ & $\mathrm{O}\left(\mathrm{M} / \mathrm{cm}^{3}\right)(\%)$ \\
\hline \hline Ammonium Nitrate & $0.084 \pm 0.002(2.3)$ & $0.000 \pm 0.000(0.0)$ & $0.051 \pm 0.003(19)$ & $0.058 \pm 0.003(11)$ \\
Graphite & $0.000 \pm 0.000(0.0)$ & $0.171 \pm 0.001(1.7)$ & $0.000 \pm 0.000(0.0)$ & $0.000 \pm 0.000(0.0)$ \\
HDPE & $0.131 \pm 0.003(2.2)$ & $0.068 \pm 0.003(1.5)$ & $0.000 \pm 0.000(0.0)$ & $0.000 \pm 0.000(0.0)$ \\
Hydrogen Peroxide & $0.080 \pm 0.003(5.8)$ & $0.010 \pm 0.004(\mathrm{n} / \mathrm{a})$ & $0.000 \pm 0.000(0.0)$ & $0.079 \pm 0.003(1.2)$ \\
Water & $0.108 \pm 0.002(2.7)$ & $0.000 \pm 0.000(0.0)$ & $0.000 \pm 0.000(0.0)$ & $0.058 \pm 0.002(7.1)$ \\
\hline
\end{tabular}

Table 4: The reconstructed molar concentrations for all samples studied with both position response and $\sigma_{t}=1.0 \mathrm{~s}$ timing response applied (and no source scattering background). The dwell time for each case is two hours. The percent error from the true value is quoted in parentheses. The parameter errors are one standard deviation, from the error matrix of MINUIT.

\begin{tabular}{|c||c|}
\hline Dwell Time (s) & $\mathrm{C}\left(\mathrm{M} / \mathrm{cm}^{3}\right)(\%)$ \\
\hline 60 & $0.171 \pm 0.009(1.7)$ \\
300 & $0.176 \pm 0.004(1.2)$ \\
890 & $0.176 \pm 0.002(1.2)$ \\
1800 & $0.175 \pm 0.002(0.6)$ \\
3600 & $0.173 \pm 0.001(0.6)$ \\
7200 & $0.171 \pm 0.001(1.7)$ \\
\hline
\end{tabular}

Table 5: The reconstructed molar concentrations for a $2 \times 30.81 \times 30.81 \mathrm{~cm}$ graphite sample as a function of dwell time in seconds, with both position response and $\sigma_{t}=1.0 \mathrm{~s}$ timing response applied (and no source scattering background) The percent error from the true value is quoted in parentheses. The parameter errors are one standard deviation, from the error matrix of MINUIT. 
The graphite sample is also used to study the effects of acquisition time and timing resolution. Table 5 shows the reconstructed molar fraction of the graphite sample with $1.0 \mathrm{~ns}$ timing and position response applied, and for a range of acquisition times in half hour increments. For these results, all parameters other than carbon were fixed to zero so that the precision and accuracy of the reconstruction were not effected by parameter error fluctuations that could effect the error on carbon. The results indicate that after one minute, the precision is $5 \%$ of the reconstructed parameter value, then drops steadily, reaching $0.6 \%$ of the reconstructed value by one hour of acquisition time. The percent error appears steady within the error of the parameter. Table 6 shows the effects of both position and timing response on the graphite sample: the accuracy of the carbon molar concentration increases to $20 \%$ for a $\sigma_{t}=2.5 \mathrm{~ns}$ timing response and position response. Finally, the effects of the sample scattering background are included in Table 7. Compared to Table 4, the accuracy has increased from less than $10 \%$ for most elements and samples to the mid-twenty percent level for most cases. This level is expected to worsen for larger samples, unless the scattered neutrons are used in the reconstruction in the manner described in Section 3.

Of particular interest for explosive detection is how well hydrogen peroxide and water can be distinguished. Figure 9 demonstrates that, even with the sample scattering background included, with a timing response of $\sigma_{t}=1 \mathrm{~ns}$ and the position response sampled from the Monte Carlo, the accuracy is sufficient to distinguish a pure hydrogen peroxide sample from a water sample, as well as characterize a range of other materials that were included in this study. Although the accuracy is as high as $30 \%$ in some cases, most of the values are systematically low. In the case of water and hydrogen peroxide, the ratio of oxygen to hydrogen is reconstructed to within $8 \pm 0.4 \%$ of the true value for water, and $3 \pm$ $0.5 \%$ for hydrogen peroxide.

\section{Conclusions and Future Work}

This work presents the expected performance of a material characterization system based on fast neutron attenuation measurements. Our results, based on a Geant4 application with detector response included, provides evidence that a system would be able to distin- ${ }^{482}$ guish pure hydrogen peroxide from water, and also successfully characterize a range of other materials based ${ }_{483}$ on the reconstruction of the molar concentration of hy- 484 drogen, carbon, nitrogen, and oxygen. Without account- 485 ing for the sample scattering background, the ratio of ${ }_{486}$ a)

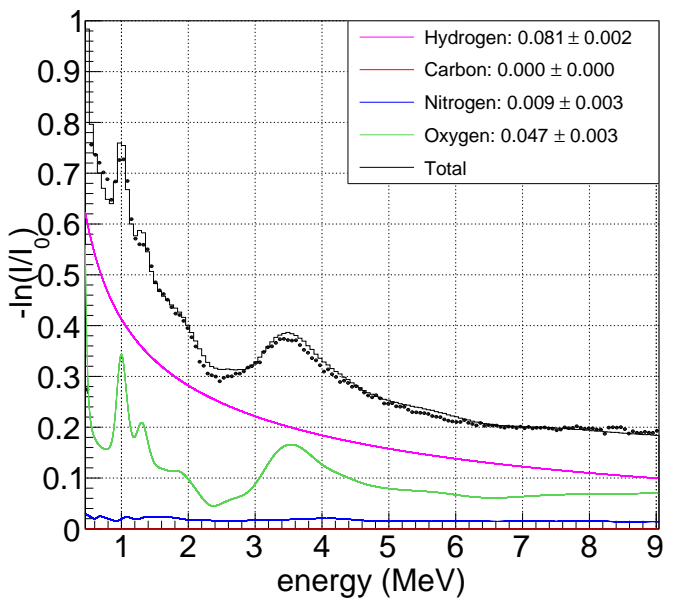

b)

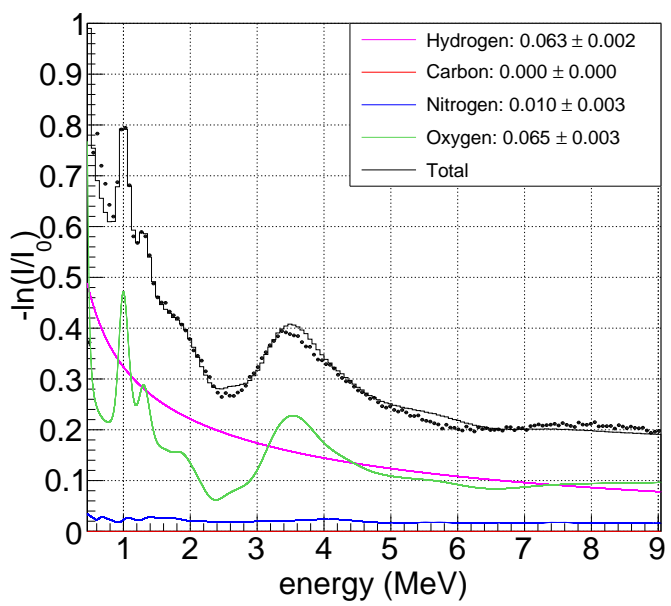

Figure 9: The attenuated energy spectrum for a $2 \times 30.48 \times 30.48$ a) water and b) hydrogen peroxide sample, including the sample scattering background and with the expected position and $\sigma_{t}=1$ ns timing response response applied. the true value. The precision of the ratio is $0.5 \%$ or less.

Future work will include validation of these results with experimental data on a range of materials. Once benchmarked, we plan to optimize the design of a larger system with the goal of reducing scan times.

\section{Acknowledgments}

This work was supported by Laboratory Directed Research and Development (LDRD) at Sandia National Laboratories. Sandia National Laboratories is a multiprogram laboratory managed and operated by Sandia 


\begin{tabular}{|c|c||c|c|c|c|}
\hline$\sigma_{t}(s)$ & $\sigma_{d}(\mathrm{~cm})$ & $\mathrm{H}\left(\mathrm{M} / \mathrm{cm}^{3}\right)(\%)$ & $\mathrm{C}\left(\mathrm{M} / \mathrm{cm}^{3}\right)(\%)$ & $\mathrm{N}\left(\mathrm{M} / \mathrm{cm}^{3}\right)(\%)$ & $\mathrm{O}\left(\mathrm{M} / \mathrm{cm}^{3}\right)(\%)$ \\
\hline \hline 0 & 0 & $0.000 \pm 0.000(0.0)$ & $0.142 \pm 0.003(18)$ & $0.024 \pm 0.003(\mathrm{n} / \mathrm{a})$ & $0.008 \pm 0.002(\mathrm{n} / \mathrm{a})$ \\
\hline 0 & $f(E)$ & $0.000 \pm 0.000(0.0)$ & $0.172 \pm 0.001(1.1)$ & $0.000 \pm 0.000(0.0)$ & $0.000 \pm 0.000(0.0)$ \\
\hline 0.5 & $f(E)$ & $0.011 \pm 0.002(\mathrm{n} / \mathrm{a})$ & $0.149 \pm 0.004(14)$ & $0.000 \pm 0.000(0.0)$ & $0.006 \pm 0.003(\mathrm{n} / \mathrm{a})$ \\
1.0 & $f(E)$ & $0.000 \pm 0.000(0.0)$ & $0.171 \pm 0.001(1.7)$ & $0.000 \pm 0.000(0.0)$ & $0.000 \pm 0.000(0.0)$ \\
2.5 & $f(E)$ & $0.014 \pm 0.003(\mathrm{n} / \mathrm{a})$ & $0.141 \pm 0.006(19)$ & $0.013 \pm 0.005(\mathrm{n} / \mathrm{a})$ & $0.000 \pm 0.000(0.0)$ \\
\hline
\end{tabular}

Table 6: The reconstructed molar concentrations for a $2 \times 30.48 \times 30.48 \mathrm{~cm}$ graphite sample, with a molar concentration of $0.174 \mathrm{M} / \mathrm{cm}^{3}$ of carbon alone. For each case the position and timing resolution of the detector units are changed in the event processing. There is no sample scattering background included, and the dwell time for each case is two hours. The parameter errors are one standard deviation, from the error matrix of MINUIT.

\begin{tabular}{|l||c|c|c|c|}
\hline Sample & $\mathrm{H}\left(\mathrm{M} / \mathrm{cm}^{3}\right)(\%)$ & $\mathrm{C}\left(\mathrm{M} / \mathrm{cm}^{3}\right)(\%)$ & $\mathrm{N}\left(\mathrm{M} / \mathrm{cm}^{3}\right)(\%)$ & $\mathrm{O}\left(\mathrm{M} / \mathrm{cm}^{3}\right)(\%)$ \\
\hline \hline Ammonium Nitrate & $0.064 \pm 0.003(26)$ & $0.007 \pm 0.006(\mathrm{n} / \mathrm{a})$ & $0.045 \pm 0.005(4.7)$ & $0.044 \pm 0.003(32)$ \\
Graphite & $0.015 \pm 0.002(\mathrm{n} / \mathrm{a})$ & $0.120 \pm 0.005(31)$ & $0.034 \pm 0.005(\mathrm{n} / \mathrm{a})$ & $0.000 \pm 0.000(0.0)$ \\
HDPE & $0.100 \pm 0.003(25)$ & $0.055 \pm 0.005(18)$ & $0.007 \pm 0.005(\mathrm{n} / \mathrm{a})$ & $0.000 \pm 0.000(0.0)$ \\
Hydrogen Peroxide & $0.063 \pm 0.002(26)$ & $0.000 \pm 0.000(0.0)$ & $0.010 \pm 0.003(\mathrm{n} / \mathrm{a})$ & $0.065 \pm 0.003(24)$ \\
Water & $0.081 \pm 0.002(27)$ & $0.000 \pm 0.000(0.0)$ & $0.009 \pm 0.003(\mathrm{n} / \mathrm{a})$ & $0.047 \pm 0.003(16)$ \\
\hline
\end{tabular}

Table 7: The reconstructed molar concentrations for all samples studied, with $\sigma_{t}=1.0 \mathrm{~s}$ and detector smearing, including the sample scattering background. The dwell time for each case is two hours. The percent error from the true value is quoted in parentheses. The parameter errors are from the error matrix of MINUIT.

Corporation, a wholly owned subsidiary of Lockheed ${ }_{520}$ Martin Corporation, for the U.S. Department of En- ${ }^{521}$ ergy's National Nuclear Security Administration un- ${ }_{522}$ der contract DE-AC04-94AL85000. Document release ${ }_{524}^{523}$ number SAND2016-6250 J.

\section{References}

[1] A. Buffler. "Contraband detection with fast neutrons." Radiation Physics and Chemistry 71 (2004) 853-861

[2] I. Mor et al. "High spatial resolution fast-neutron imaging detectors for Pulsed Fast-Neutron Transmission Spectroscopy" Journal of Instrumentation 4 (2009) P05016

[3] I. Mor et al. "Parameters affecting image quality with TimeResolved Optical Integrative Neutron (TRION) detector" Nuclear Instruments and Methods in Physics Research A 640 (2011) 192199

[4] G. Chen and R. Lanza. "Fast Neutron Resonance Radiography for Elemental Imaging: Theory and Applications" IEEE Transactions on Nuclear Science 49 (2002) 1919-1924

[5] J. Rahon, A. Danagoulian, T. D. MacDonald, Z. S. Hartwig, and R. Lanza. "Spectroscopic neutron radiography for a cargo scanning system" Nuclear Instruments and Methods in Physics Research A 820 (2016) 141-145

[6] B. D. Sowerby et al. "Recent Developments in Fast Neutron Radiography for the Interrogation of Air Cargo Containers" IAEA Conference, 4-8 May 2009

[7] M.B. Chadwick et al. "Next generation evaluated nuclear data library for nuclear science and technology." Nuclear Data Sheets 107 (2006) 2931

[8] P. Marleau et al. "Fast Neutron Resonance Tomography using Double Scatter Spectroscopy for Materials Identification" IEEE NSS conference proceedings (2011)

[9] J. Allison et al. "Geant4 developments and applications" IEEE Transactions on Nuclear Science 53 (2006) 270-278
[10] http:my.et-enterprises.com/pdf/9821B.pdf, accessed on October 21, 2015.

[11] R. Brun and F. Rademakers. Nucl. Inst. and Meth. in Phys. Res. A 389 (1997) 81-86

[12] F. James and M. Winkler "MINUIT User's Guide" http://lcgapp.cern.ch/project/cls/workpackages/mathlibs/minuit/doc/doc.html, Accessed on February 4, 2016

[13] F. James and M. Roos. Computer Physics Communications 10 (1975) 343-367. 